Pacific Journal of Mathematic

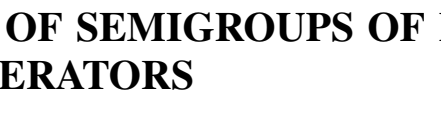




\title{
ON THE GENERATION OF SEMIGROUPS OF LINEAR OPERATORS
}

\author{
R. S. Phillips
}

1. Introduction. Let $T(\xi)$ be a semigroup of linear bounded transformations on a Banach space $\mathfrak{X}$ to itself (see [4]), strongly continuous on $[0, \infty)$ with $T(0)=I$. Further let $\alpha(t, \xi)$ be a one-parameter family of functions of bounded variation in $\xi \geq 0$ which form a semigroup in $t \geq 0$ with product defined by convolution. Then

$$
S(t)=\int_{0}^{\infty} T(\xi) d_{\xi} \alpha(t, \xi)
$$

will also form a semigroup of linear bounded transformations on $\mathscr{X}$ to itself. If the semigroup $\alpha(t, \cdot)$ satisfies certain continuity conditions, then $S(t)$ will be strongly continuous for $t \geq 0$. This method of generating new semigroups out of old ones has previously been considered in a general way by N. P. Roman off [10] and in connection with stochastic processes by S. Bochner [2]. We shall consider the problem in the setting described above and attempt to obtain the infinitesimal generator $B$ of the semigroup $S(t)$ directly in terms of the semigroup $T(\xi)$ and its infinitesimal generator $A$. We shall seek also to relate the spectrum of $B$ to that of $A$.

In general the integral in (1) will not converge unless $\alpha$ is suitably restricted. The most general function $\alpha$ of bounded variation, for which the integral converges absolutely, will satisfy the condition

$$
\int_{0}^{\infty} \exp [\omega(\xi)]|d \alpha|<\infty
$$

where $\omega(\xi) \equiv \log \|T(\xi)\|$ is lower semicontinuous, subadditive, and bounded near the origin. We shall accordingly limit ourselves to the Banach algebra $\mathcal{S}(\omega)$ consisting of the set of all such functions $\alpha$, where the norm is given by the integral in (2) and the product is given by convolution. Now $\subseteq(\omega)$ can also be considered as an operator algebra over the Banach space $\mathfrak{R}(\omega)$ consisting of the absolutely continuous elements in $\mathscr{S}(\omega)$. In the course of proving that the operator topology for $E(\omega)$ and the original topology are isomorphic we have

Received January 29, 1952.

Pacific J. Math. 2 (1952), 343-369 
obtained the following relation for the case $\lim _{\xi \rightarrow 0} \omega(\xi)=0$ :

$$
\int_{0}^{\infty} \exp [\omega(\xi)]|d \alpha|=\lim _{\delta \rightarrow 0} \int_{0}^{\infty} \exp [\omega(\xi)]\left|\frac{\alpha(\xi)-\alpha(\xi-\delta)}{\delta}\right| d \xi
$$

We have been able to extend the results of A. Kolmogoroff [7] and Paul Lévy [8] on semigroups of distribution functions to the case of semigroups of monotonic nondecreasing functions in $G(\omega)$ when $\omega(\xi)$ satisfies the additional condition

$$
\lim _{\delta \rightarrow 0}\left(\sup _{\xi \geq 0}[\omega(\xi)-\omega(\xi+\delta)]\right)<\infty
$$

In fact, if we set $\omega_{0}=\inf \omega(\xi) / \xi$ and

$$
\Phi[\lambda, \alpha]=\int_{0}^{\infty} \exp [\lambda \xi] d \alpha \quad \text { for } R[\lambda] \leq \omega_{0}
$$

then we have shown that a necessary and sufficient condicion that $\alpha(t, \xi)$ be a semigroup of monotone functions in $\mathcal{S}(\omega)$ (strongly continuous over $\Omega(\omega)$ at $t=0)$ is that

$$
t^{-1} \log \Phi[\lambda, \alpha(t, \cdot)]=m \lambda+\int_{0}^{\infty}\left(\exp \left[\left(\lambda-\omega_{0}\right) \xi\right]-1\right) d \psi+a
$$

where $m \geq 0, a$ is real, and $\psi$ is monotonic nondecreasing on $(0, \infty)$ satisfying the conditions

$$
\int_{0}^{1} \xi d \psi<\infty \text { and } \int_{1}^{\infty} \exp \left[\omega(\xi)-\omega_{0} \xi\right] d \psi<\infty
$$

Finally, we have been able to obtain a characterization of the infinitesimal generator $B$ of $S(t)$ when $S(t)$ is generated by a semigroup of monotone functions in $\subseteq(\omega)$. In this case we show that for $x$ in the domain of $A$ we have

$$
B x=m A x+\int_{0}^{\infty}\left[\exp \left(-\omega_{0} \xi\right) T(\xi) x-x\right] d \psi+a x
$$

where $m, a$, and $\psi(\xi)$ are obtained from (6). In addition, if $\lambda_{0}$ belongs to the spectrum of $A$, then

$$
\left.m \lambda_{0}+\int_{0}^{\infty}\left(\exp \left[\lambda_{0}-\omega_{0}\right) \xi\right]-1\right) d \psi+a
$$

belongs to the spectrum of $B$. 
2. The Banach algebra $S(\omega)$. This section will be devoted to a characterization of various kinds of convergence in the Banach algebra $\mathfrak{S}(\omega)$. We shall suppose that the weight function $\omega(\xi)$ satisfies the hypothesis

(i) $\omega(\xi)$ is real valued and lower semicontinuous on $[0, \infty)$;

(h) (ii) $\omega(0)=0, \overline{\lim } \omega(\xi)<\infty$;

(iii) $\omega(\xi)$ is subad ditive: $\omega\left(\xi_{1}+\xi_{2}\right) \leq \omega\left(\xi_{1}\right)+\omega\left(\xi_{2}\right)$ for $\xi_{1}, \xi_{2} \geq 0$. If, in addition,

$$
\text { (iv) } \lim _{\delta \rightarrow 0+}\left[\sup _{\xi \geq 0}(\omega(\xi)-\omega(\xi+\delta))\right]<\infty,
$$

we shall say that $\omega$ satisfies the hypothesis $\left(h^{*}\right)$. Conditions (i) and (iii) imply (see [4, Chap. 6]) that $\underline{\lim }_{\xi \rightarrow 0^{+}} \omega(\xi) \geq 0$, and hence that $\omega(\xi)$ is bounded in every finite interval $[0, L]$. Further,

$$
\omega_{0}=\inf _{\xi>0} \omega(\xi) / \xi=\lim _{\xi \rightarrow \infty} \omega(\xi) / \xi
$$

We now define $\mathfrak{S}(\omega)$ to be the set of all completely additive complex-valued set functions $\alpha(\sigma)$ on the sigma-field of Borel measurable subsets $B$ of $[0, \infty)$ such that

$$
\int_{0}^{\infty} \exp [\omega(\xi)]|d \alpha|<\infty
$$

We require only that $\alpha(\sigma)$ be finite if $\sigma$ is contained in a finite interval. The norm is given by

$$
\|\alpha\|=\int_{0}^{\infty} \exp [\omega(\xi)]|d \alpha|
$$

It is clear that $\widetilde{S}(\omega)$ is a Banach space. In order to define the product $\gamma=\alpha * \beta$ of two elements of $\subseteq(\omega)$, we consider the product measure $\bar{\gamma}$ of $\alpha$ and $\beta$ defined on the smallest sigma-field $B_{2}$ generated by $B \times B$. For any $\sigma \in \mathfrak{B}$, we set

$$
\gamma(\sigma) \equiv \bar{\gamma}[(u, v) \mid u+v \in \sigma ; u, v \geq 0]
$$

It follows that the product is commutative, that

$$
\gamma(\sigma)=\int_{0}^{\infty} \alpha(\sigma-u) d_{u} \beta=\int_{0}^{\infty} \beta(\sigma-u) d_{u} \alpha
$$


and that

$$
\|\gamma\| \leq\|\alpha\| \cdot \| \beta H \cdot
$$

Thus $\mathfrak{S}(\omega)$ is likewise a Banach algebra with unit $e$. It will sometimes be convenient to consider $\alpha \in G(\omega)$ not as a set function $\alpha(\sigma)$, but simply as a function of bounded variation continuous on the left, namely as

$$
\alpha(\xi) \equiv \alpha([0, \xi))
$$

This correspondence between set function and point function is clearly one-toone and should not cause any confusion.

Let $\mathfrak{R}(\omega)$ be the set of Borel measurable functions $f(\xi)$ such that

$$
\int_{0}^{\infty} \exp [\omega(\xi)]|f(\xi)| d \xi<\infty
$$

Then with norm

$$
\|f\|=\int_{0}^{\infty} \exp [\omega(\xi)]|f(\xi)| d \xi
$$

$\mathcal{l}(\omega)$ is a Banach space. Further if $\alpha \in \subseteq(\omega)$, then

$$
A_{\alpha}(f)=\int_{0}^{\infty} f(\xi-u) d_{u} \alpha
$$

defines a linear bounded transformation on $\{(\omega)$ to itself. Clearly

$$
\left\|A_{a}(f)\right\| \leq\|\alpha\|\|f\|
$$

and hence

$$
\left\|A_{\alpha}\right\| \leq\|\alpha\|
$$

We shall show that the two norms are isomorphic. To this end we set $f_{\delta}(\xi)=$ $1 / \delta$ for $0<\xi \leq \delta$ and zero elsewhere. Then by (14),

$$
\begin{aligned}
\varlimsup_{\delta \rightarrow 0+} \int_{0}^{\infty} \exp [\omega(\xi)]\left|\frac{\alpha(\xi)-\alpha(\xi-\delta)}{\delta}\right| d \xi \\
=\varlimsup_{\delta \rightarrow 0+}\left\|A_{\alpha}\left(f_{\delta}\right)\right\| \leq c\|\alpha\|,
\end{aligned}
$$

where 


$$
c=\varlimsup_{\delta \rightarrow 0^{+}} \delta^{-1} \int_{0}^{\delta} \exp [\omega(\xi)] d \xi
$$

Because of $(\mathrm{h}-\mathrm{ii})$ and the subsequent remarks, it is clear that $l \leq c<\infty$.

Leмma 2.1. If $\omega(\xi)$ is subadditive and merely lower semicontinuous, then

$$
\varliminf_{\delta \rightarrow 0_{+}} \int_{0}^{\infty} \exp [\omega(\xi)]\left|\frac{\alpha(\xi)-\alpha(\xi-\delta)}{\delta}\right| d \xi \geq\|\alpha\| .
$$

Since $\exp [\omega(\xi)]$ is lower semicontinuous, we can approximate it from below by a sequence of continuous functions $w_{n}(\xi)$ such that

$$
0 \leq w_{n}(\xi) \leq w_{n+1}(\xi) \text { and } \lim _{n \rightarrow \infty} w_{n}(\xi)=\exp [\omega(\xi)]
$$

pointwise. We shall show that

$$
\lim _{\delta \rightarrow 0+} \int_{0}^{\infty} w_{n}(\xi)\left|\frac{\alpha(\xi)-\alpha(\xi-\delta)}{\delta}\right| d \xi \geq \int_{0}^{\infty} w_{n}(\xi)|d \alpha|,
$$

and hence that

$$
\varliminf_{\delta \rightarrow 0^{+}} \int_{0}^{\infty} \exp [\omega(\xi)]\left|\frac{\alpha(\xi)-\alpha(\xi-\delta)}{\delta}\right| d \xi \geq \int_{0}^{\infty} w_{n}(\xi)|d \alpha|
$$

for all $n$. The inequality (17) then follows by Fatou's lemma. It remains to prove (18). Now given $\epsilon>0$ there exists a subdivision $\xi_{0}=0<\xi_{1}<\cdots<\xi_{n}=L$ such that

$$
\int_{0}^{\infty} w(\xi)|d \alpha|<\sum_{i=1}^{h} w\left(\xi_{i}^{\prime}\right)\left|\alpha\left(\xi_{i}\right)-\alpha\left(\xi_{i-1}\right)\right|+\epsilon,
$$

where $\xi_{i-1} \leq \xi_{i}^{\prime} \leq \xi_{i}$; and this inequality remains true for all refinements of the above subdivision of $[0, L]$. Setting

$$
W(\xi)=\int_{a}^{\xi} w(u) d u
$$

we have 


$$
\begin{array}{rl}
\int_{a}^{b} & w(\xi)[\alpha(\xi)-\alpha(\xi-\delta)] d \xi \\
\quad=\left.W(\xi)[\alpha(\xi)-\alpha(\xi-\delta)]\right|_{a} ^{b}-\int_{a}^{b} W(\xi) d \alpha(\xi)+\int_{a}^{b} \mathbb{W}(\xi) d \alpha(\xi-\delta)
\end{array}
$$

$$
\begin{aligned}
=\int_{b-\delta}^{b}[W(b)-W(\xi+\delta)] d \alpha-\int_{a-\delta}^{a}[W(a) & W(\xi+\delta)] d \alpha \\
& +\int_{a}^{b}[W(\xi+\delta)-W(\xi)] d \alpha .
\end{aligned}
$$

Now

$$
\begin{aligned}
\mid \int_{a}^{b}[\mathbb{W}(\xi+\delta)-W(\xi)] d \alpha-w & \left(\xi^{\prime}\right) \delta[\alpha(b)-\alpha(a)] \mid \\
& \leq \operatorname{osc}[w ; a, b+\delta] \operatorname{var}[\alpha ; a, b]
\end{aligned}
$$

where $a \leq \xi^{\prime} \leq b$. If $M=\max [|w(\xi)| \mid \xi \in[0, L]]$, we see that the first two terms on the right of $(20)$ are bounded above by $M \delta \operatorname{var}[\alpha ; b-\delta, b]$ and by $M \delta \operatorname{var}[\alpha ; a-\delta, a]$ respectively. Hence

$$
\begin{aligned}
\sum_{i=1}^{n} \int_{\xi_{i-1}}^{\xi_{i}} w(\xi)\left|\frac{\alpha(\xi)-\alpha(\xi-\delta)}{\delta}\right| d \xi & \\
& \geq \sum_{i=1}^{n}\left|\int_{\xi_{i-1}}^{\xi_{i}} w(\xi) \frac{\alpha(\xi)-\alpha(\xi-\delta)}{\delta} d \xi\right| \\
& \geq \sum_{i=1}^{n} w\left(\xi_{i}^{\prime}\right)\left|\alpha\left(\xi_{i}\right)-\alpha\left(\xi_{i-1}\right)\right| \\
& -\sum_{i=1}^{n} \operatorname{osc}\left[w ; \xi_{i-1}, \xi_{i}+\delta\right] \operatorname{var}\left[\alpha ; \xi_{i-1}, \xi_{i}\right] \\
& -M \sum_{i=1}^{n}\left(\operatorname{var}\left[\alpha ; \xi_{i-1}-\delta, \xi_{i-1}\right]+\operatorname{var}\left[\alpha ; \xi_{i}-\delta, \xi_{i}\right]\right) .
\end{aligned}
$$

We first choose a subdivision sufficiently fine so that for all sufficiently small $\delta>0$ we have 


$$
\max _{i}\left\{\operatorname{osc}\left[w ; \xi_{i-1}, \xi_{i}+\delta\right]\right\}<\frac{\epsilon}{\operatorname{var}[\alpha ; 0, L]}
$$

Combining (19) with the above inequality we then get

$$
\begin{array}{r}
\int_{0}^{\infty} w(\xi)|d \alpha|<\int_{0}^{\infty} w(\xi)\left|\frac{\alpha(\xi)-\alpha(\xi-\delta)}{\delta}\right| d \xi \\
+2 \epsilon+2 M \sum_{i=0}^{n} \operatorname{var}\left[\alpha ; \xi_{i}-\delta, \xi_{i}\right]
\end{array}
$$

The inequality (18) now follows from the fact that $\alpha$ is continuous on the left and the arbitrariness of $\epsilon>0$.

As a consequence of Lemma 2.1 we see that

$$
\left\|A_{\alpha}\right\|=\sup \left\|A_{\alpha}(f)\right\| /\|f\| \geq \lim _{\delta \rightarrow 0^{+}}\left\|A_{\alpha}\left(f_{\delta}\right)\right\| / \varlimsup_{\delta \rightarrow 0^{+}}\left\|f_{\delta}\right\| \geq\|\alpha\| / c .
$$

Combining this with (14) we have

$$
\|\alpha\| / c \leq\left\|A_{\alpha}\right\| \leq\|\alpha\| \text {. }
$$

THEOREM 2.1. The operator norm for the elements of $\mathcal{S}(\omega)$ over $\Omega(\omega)$ is isomorphic to the regular norm (9).

In particular, if $\lim _{\xi \rightarrow 0+} \omega(\xi)=0$ then $c=1$, and hence (3) follows from (15) and (17).

We next obtain a criterion for the strong convergence of elements in $\subseteq(\omega)$ considered as operators on $\mathfrak{R}(\omega)$.

THEOREM 2.2. The operators $A_{a_{n}}$ converge strongly to 0 if and only if the $\left\|\alpha_{n}\right\|$ are bounded and

$$
\lim _{n \rightarrow \infty} \int_{0}^{\infty} \exp [\omega(\xi)]\left|\alpha_{n}(\xi)-\alpha_{n}(\xi-\delta)\right| d \xi=0
$$

for all sufficiently small $\delta>0$.

If $\lim _{n \rightarrow \infty} A_{a_{n}}(f)=0$ for each $f \in\{(\omega)$, then by a well-known theorem due to Banach $[1, \mathrm{p} .80]$ the $\left\|A_{a_{n}}\right\|$ are bounded, and hence by Theorem 2.1 so are the $\left\|\alpha_{n}\right\|$. Further, if $f_{\delta}(\xi)=1$ for $0<\xi \leq \delta$ and zero elsewhere, then 


$$
\left\|A_{\alpha_{n}}\left(f_{\delta}\right)\right\|=\int_{0}^{\infty} \exp [\omega(\xi)]\left|\alpha_{n}(\xi)-\alpha_{n}(\xi-\delta)\right| d \xi \rightarrow 0
$$

for all $\delta>0$. Conversely, suppose (22) is valid for all $\delta \in\left(0, \delta_{0}\right)$. Then given $a>0$ there exists an integer $k$ and a $\delta \in\left(0, \delta_{0}\right)$ such that $k \delta=a$. Now for any $\xi_{0} \geq 0$ we have

$$
\begin{aligned}
& \int_{0}^{\infty} \exp [\omega(\xi)]\left|\alpha_{n}\left(\xi-\xi_{0}\right)-\alpha_{n}\left(\xi-\xi_{0}-\delta\right)\right| d \xi \\
& \leq \exp \left[\omega\left(\xi_{0}\right)\right] \int_{0}^{\infty} \exp [\omega(\xi)]\left|\alpha_{n}(\xi)-\alpha_{n}(\xi-\delta)\right| d \xi \rightarrow 0
\end{aligned}
$$

as $n \rightarrow \infty$. Hence

$$
\begin{aligned}
\int_{0}^{\infty} \exp [\omega(\xi)]\left|\alpha_{n}(\xi)-\alpha_{n}(\xi-a)\right| d \xi \\
\quad \leq \sum_{j=1}^{k} \int_{0}^{\infty} \exp [\omega(\xi)]\left|\alpha_{n}(\xi-(j-1) \delta)-\alpha_{n}(\xi-j \delta)\right| d \xi \rightarrow 0
\end{aligned}
$$

as $n \rightarrow \infty$. In other words, if $f(\xi)=1$ for $0<\xi \leq a$ and zero elsewhere, then $\left\|A_{a_{n}}(f)\right\| \rightarrow 0$. Since the linear extension of this class of functions is dense in $\mathcal{R}(\omega)$, and since $\left\|A_{a_{n}}\right\|$ are bounded by (14), the desired result follows from the Banach-Steinhaus theorem [1, p. 79].

We now define

$$
\Phi(\lambda, \alpha)=\int_{0}^{\infty} \exp [\lambda \xi] d \alpha
$$

$$
\phi(\lambda, f)=\int_{0}^{\infty} \exp [\lambda \xi] f(\xi) d \xi
$$

These integrals converge absolutely for $R[\lambda] \leq \omega_{0}$. We recall that the Laplace transform of the convolution of two functions is the product of their Laplace transforms, and hence that

$$
\int_{0}^{\infty} \exp [\lambda \xi] A_{a}(f) d \xi=\Phi(\lambda) \phi(\lambda)
$$

We next obtain a necessary condition for the strong convergence of the operators $A_{\alpha_{n}}$, where again $\alpha_{n} \in \mathbb{S}(\omega)$. 
THEOREM 2.3. If the operators $A_{a_{n}}$ converge to 0 in the strong topology, then the $\left\|\alpha_{n}\right\|$ are bounded, $\Phi_{n}(\lambda) \rightarrow 0$ uniformly in every bounded subset of $R[\lambda] \leq \omega_{0}$, and $\alpha_{n}(\xi) \rightarrow 0$ pointwise.

The boundedness of the $\left\|\alpha_{n}\right\|$ follows as in Theorem 2.2. Since

$$
|\phi(\lambda, f)| \leq\|f\| \text { for } R[\lambda] \leq \omega_{0},
$$

it follows from (24) that $\left|\Phi_{n}(\lambda) \phi(\lambda, f)\right| \leq\left\|A_{a_{n}}(f)\right\| \rightarrow 0$ uniformly for $R[\lambda] \leq \omega_{0}$. The uniform convergence of $\Phi_{n}(\lambda)$ to zero on a bounded subset $E$ of $R[\lambda] \leq \omega_{0}$ now follows from the fact that there exist functions $\phi(\lambda, f)$ bounded away from zero on $E$. In fact, for $f_{\delta}$ defined as in the previous theorem, $\phi\left(\lambda, f_{\delta}\right)=(\exp (\lambda \delta)-1) / \lambda$ will suffice for $\delta$ sufficiently small. The fact that $\alpha_{n}(\xi) \rightarrow 0$ is a consequence of the following lemma, patterned after the P. Lévy convergence theorem $[8, \mathrm{p} .49]$.

Lemma 2.2. If $\alpha_{n} \in \subseteq(\omega)$, the $\left\|\alpha_{n}\right\|$ are bounded, and $\Phi\left(\lambda, \alpha_{n}\right)$ converges to $\Omega(\lambda)$ uniformly in every bounded subset of $R[\lambda] \leq \omega_{0}$, then there exists an $\alpha \in \subseteq(\omega)$ such that $\Phi(\lambda, \alpha)=\Omega(\lambda)$ and $\alpha_{n}(\xi) \rightarrow \alpha(\xi)$ at every point of continuity of $\alpha$.

Since the variations of the $\alpha_{n}$ are uniformly bounded in every finite interval, we can apply the Helly theorem and obtain a subsequence $\alpha_{n_{k}}(\xi)$ which converges to a function $\alpha(\xi)$ (likewise of bounded variation in every finite interval and continuous on the left) at each point of continuity of $\alpha(\xi)$. In order to show that $\alpha \in \subseteq(\omega)$, we approximate $\exp [\omega(\xi)]$ as in Lemma 2.1 by a sequence of continuous functions $w_{n}(\xi)$, where

$$
0 \leq w_{n}(\xi) \leq w_{n+1}(\xi) \text { and } w_{n}(\xi) \rightarrow \exp [\omega(\xi)]
$$

pointwise. Then, by hypothesis,

$$
\int_{0}^{\infty} w_{n}(\xi)\left|d \alpha_{n_{k}}\right| \leq \int_{0}^{\infty} \exp [\omega(\xi)]\left|d \alpha_{n_{k}}\right| \leq M .
$$

For each $L>0$ we have

$$
\int_{0}^{L} w_{n}(\xi)|d \alpha| \leq \lim _{k \rightarrow \infty} \int_{0}^{L} w_{n}(\xi)\left|d \alpha_{n_{k}}\right|
$$

Hence $\int_{0}^{\infty} w_{n}(\xi)|d \alpha| \leq M$ for each $n$; and, by Fatou's lemma,

$$
\int_{0}^{\infty} \exp [\omega(\xi)]|d \alpha| \leq M
$$


Thus $\alpha \in \subseteq(\omega)$. On the other hand, for any $\delta>0$ and $u=R(\lambda) \leq \omega_{0}$, we have

$$
(2 \delta)^{-1} \int_{v-\delta}^{v+\delta} \Phi_{n}(u+i \nu) d \nu=\int_{0}^{\infty} \exp [(u+i v) \xi](\delta \xi)^{-1} \sin \delta \xi d \alpha_{n}
$$

Now

$$
\left|\int_{L}^{\infty} \exp [(u+i v) \delta](\delta \xi)^{-1} \sin \delta \xi d \alpha_{n}\right| \leq M /(\delta L)
$$

so that

$$
\begin{aligned}
\int_{0}^{\infty} \exp [(u+i v) \xi] & (\delta \xi)^{-1} \sin \delta \xi d \alpha_{n} \\
& \rightarrow \int_{0}^{\infty} \exp [(u+i v) \xi](\delta \xi)^{-1} \sin \delta \xi d \alpha
\end{aligned}
$$

Finally, since $\Phi_{n}(\lambda) \rightarrow \Omega(\lambda)$ uniformly in every bounded subset of $R[\lambda] \leq \omega_{0}$, we obtain, for all $\delta>0$,

$$
\begin{aligned}
(2 \delta)^{-1} \int_{v-\delta}^{v+\delta} \Omega(u+i \nu) d \nu & =\int_{0}^{\infty} \exp [(u+i v) \xi](\delta \xi)^{-1} \sin \delta \xi d \alpha \\
& =(2 \delta)^{-1} \int_{v-\delta}^{v+\delta} \Phi(u+i \nu) d \nu
\end{aligned}
$$

Thus $\Phi(\lambda) \equiv \Omega(\lambda)$ for $R[\lambda] \leq \omega_{0}$. Finally since this is true of all subsesequences, it follows from the uniqueness theorem for Laplace transforms that $\alpha_{n}(\xi) \rightarrow \alpha(\xi)$ at all points of continuity of $\alpha(\xi)$.

3. Nonnegative semigroups in $\widetilde{S}(\omega)$. In this section we shall obtain a generalization of the Kolmogoroff [7] and P. Lévy [8, Chap. 7] representation for semigroups of distribution functions in terms of their characteristic functions. We shall consider the semigroup $\alpha(t, \xi)$, where

(i) for each $t \geq 0, \alpha(t, \xi)$ is a nondecreasing function in $\subseteq(\omega)$;

(ii) $\alpha\left(t_{1}+t_{2}, \cdot\right)=\alpha\left(t_{1}, \cdot\right) * \alpha\left(t_{2}, \cdot\right), \alpha(0, \cdot)=e$;

(25) (iii) there exists an $M$ such that $\|\alpha(t, \cdot)\| \leq M$ for $0 \leq t \leq 1$;

(iv) $\Phi[\lambda ; \alpha(t, \cdot)]=\int_{0}^{\infty} \exp (\lambda \xi) d_{\xi} \alpha(t, \xi)$ converges to one as $t \rightarrow 0+$ uniformly in any bounded subset of $R[\lambda] \leq \omega_{0}$.

It will be convenient to introduce the auxiliary space $\mathbb{S}(\bar{\omega})$, where 


$$
\bar{\omega}(\xi)=\omega(\xi)-\omega_{0} \xi
$$

It is clear that $\bar{\omega}(\xi)$ will also satisfy the hypothesis $(\mathrm{h})$ and that inf $\bar{\omega}(\xi) / \xi=$ 0 . Hence if $\beta \in \subseteq(\bar{\omega})$, then $\beta$ is a function of bounded variation on $[0, \infty)$. The transformation

$$
\beta(\sigma) \equiv U(\alpha)(\sigma) \equiv \int_{\sigma} \exp \left(\omega_{0} \xi\right) d_{\xi} \alpha
$$

is a norm preserving isomorphism of the Banach algebra $\widetilde{S}(\omega)$ onto $\mathbb{S}(\bar{\omega})$. Further,

$$
\Phi(\lambda, \alpha)=\int_{0}^{\infty} \exp (\lambda \xi) d_{\xi} \alpha=\int_{0}^{\infty} \exp \left[\left(\lambda-\omega_{0}\right) \xi\right] d_{\xi} \beta=\Phi\left(\lambda-\omega_{0}, \beta\right)
$$

Hence if

$$
\beta(t, \cdot)=U[\alpha(t, \cdot)]
$$

then all of the conditions (25) are fulfilled for the semigroup $\beta(t, \cdot) \subset \mathcal{S}(\bar{\omega})$ with 0 replacing $\omega_{0}$ in (iv). Since, in particular,

$$
\begin{aligned}
& 0 \leq \beta(t, \infty) \leq\|\beta(t, \cdot)\| \leq M \quad \text { for } 0 \leq t \leq 1, \\
& \beta\left(t_{1}+t_{2}, \infty\right)=\beta\left(t_{1}, \infty\right) \beta\left(t_{2}, \infty\right),
\end{aligned}
$$

it follows from well-known results on multiplicative functions that $\beta(t, \infty)=$ $\exp (a t)$. We now define

$$
\gamma(t, \xi)=\exp (-a t) \beta(t, \xi)
$$

Then $\gamma(t, \cdot)$ is a semigroup of distributions, continuous in the sense of (iv). Hence we can apply the results of Kolmogoroff and P. Lévy. We refer the reader to Khintchine's proof [6] which has been reprinted by Hille [4, p. 435]. Implicit in P. Lévy's discussion of this theorem [8, p. 178] is the following modification, valid for one-sided distributions (that is, distributions defined on $[0, \infty)$ ).

Lемма 3.1. Let $\gamma(t, \xi)$ be a semigroup of one-sided distributions such that

$$
\lim _{t \rightarrow 0^{+}} \gamma(t, \xi)=1 \quad \text { for each } \xi>0 \text {. }
$$

Then

$$
t^{-1} \log (\Phi(\lambda, \gamma(t, \cdot)])=m \lambda+\int_{0}^{\infty}[\exp (\lambda \xi)-1] d \psi(\xi)
$$


for all $R(\lambda) \leq 0$, where $m \geq 0 ; \psi(\xi)$ is nondecreasing on $(0, \infty) ; \psi(\infty)<\infty$; and $\int_{0}^{1} \xi d \psi<\infty$. Conversely, every such choice of $m$ and $\psi$ defines a semigroup of one-sided distributions satisfying (28).

For the sake of completeness, we shall sketch a proof of this fact. The reader will be able to fill in the details by referring to Hille [4, pp. 435-438]. It is readily seen that

$$
\log (\Phi[\lambda, \gamma(t, \cdot)]) \equiv t \Phi_{1}(\lambda)
$$

and hence that

$$
\Phi_{1}(\lambda)=\lim _{t \rightarrow 0^{+}} t^{-1} \int_{0}^{\infty}[\exp (\lambda \xi)-1] d_{\xi} \gamma(t, \xi)
$$

We next define

$$
G(t, \xi)=t^{-1} \int_{0}^{\xi} \eta^{2} /\left(1+\eta^{2}\right) d_{\eta} \gamma(t, \eta)
$$

As in [4], it can be shown that $\operatorname{var}[G(t, \cdot)]$ is bounded for $0<t \leq t_{0}$; that the variation of $G(t, \xi)$ in $[L, \infty)$ goes to zero as $L \rightarrow \infty$, uniformly for $0<t \leq t_{0}$; and that for some sequence $t_{n}$ tending to $0+$,

$$
G\left(t_{n}, \xi\right) \rightarrow G(\xi) \text { and } m_{n}=\int_{0}^{\infty} \xi^{-1} d G\left(t_{n}, \xi\right) \rightarrow m_{0} \geq 0
$$

It follows that $G(\xi)$ can have no jump at the origin and that

$$
m_{0} \geq \lim _{n \rightarrow \infty} \int_{\epsilon}^{\infty} \xi^{-1} d G\left(t_{n}, \xi\right)=\int_{\epsilon}^{\infty} \xi^{-1} d G(\xi)
$$

Thus $\int_{0}^{\infty} \xi^{-1} d G(\xi)<\infty$. Hence, by $(30)$,

$$
\begin{aligned}
\Phi_{1}(\lambda)= & \lim _{n \rightarrow \infty}\left\{m_{n} \lambda\right. \\
& \left.+\int_{0}^{\infty}\left[\exp (\lambda \xi)-1-\lambda \xi /\left(1+\xi^{2}\right)\right]\left[\left(1+\xi^{2}\right) / \xi^{2}\right] d G\left(t_{n}, \xi\right)\right\} \\
= & m \lambda+\int_{0}^{\infty}[\exp (\lambda \xi)-1] d \psi(\xi),
\end{aligned}
$$

where 


$$
m=m_{0}-\int_{0}^{\infty} \xi^{-1} d G(\xi) \geq 0 \text { and } \psi(\xi)=-\int_{\xi}^{\infty}\left(1+\eta^{2}\right) / \eta^{2} d G(\eta)
$$

Thus the function $\psi(\xi)$ is monotonic nondecreasing on $(0, \infty) ; \psi(\xi) \leq 0$; and $\int_{0}^{1} \xi d \psi(\xi)<\infty$. The uniqueness of $\psi(\xi)$ follows from the uniqueness of $G(\xi)$ as in [4]; the converse statement is also proved as in [4].

For our purposes we shall need a theorem of this type applicable to semigroups in $\mathcal{S}(\bar{\omega})$ satisfying the conditions of (25). We shall show that if

$$
\int_{1}^{\infty} \exp [\bar{\omega}(\xi)] d \psi(\xi)<\infty
$$

then $\gamma(t, \xi) \in \subseteq(\bar{\omega})$, and that the converse is likewise true for suitable restricted $\bar{\omega}(\xi)$. Without loss of generality we set $t=1$.

THEOREм 3.1. If

$$
\log \Phi(\lambda, \gamma)=m \lambda+\int_{0}^{\infty}[\exp (\lambda \xi)-1] d \psi(\xi),
$$

where

$$
m \geq 0, \int_{0}^{1} \xi d \psi<\infty \text {, and } \int_{1}^{\infty} \exp [\bar{\omega}(\xi)] d \psi(\xi)<\infty \text {, }
$$

then $\gamma \in \subseteq(\bar{\omega})$.

It is clear that $\gamma$ is the convolution of three one-sided distributions, namely:

$$
\begin{aligned}
& \gamma_{1}=\text { an } m-\text { shift to the right, } \\
& \gamma_{2} \text {, where } \log \Phi\left(\lambda, \gamma_{2}\right)=\int_{0}^{1}[\exp (\lambda \xi)-1] d \psi, \\
& \gamma_{3}, \text { where } \log \Phi\left(\lambda, \gamma_{3}\right)=\int_{1}^{\infty}[\exp (\lambda \xi)-1] d \psi .
\end{aligned}
$$

It is further clear that $\gamma_{1} \in \mathscr{S}(\bar{\omega})$, so that it remains to show that the same is true of $\gamma_{2}$ and $\gamma_{3}$. Now $\int_{0}^{1}[\exp (\lambda \xi)-1] d \psi(\xi)$ can be approximated by sums of the type

$$
\sum_{i=1}^{n}\left[\exp \left(\lambda \xi_{i}\right)-1\right] \Delta_{i} \psi
$$

uniformly in every bounded subset of $R(\lambda) \leq 0$. Suppose $\gamma_{\pi}$ is such that

$$
\log \Phi\left(\lambda, \gamma_{\pi}\right)=\sum_{i=1}^{n}\left[\exp \left(\lambda \xi_{i}\right)-1\right] \Delta_{i} \psi
$$


Then $\gamma_{\pi}$ is the convolution of $n$ Poisson distributions, and hence has a jump of

$$
\exp \left(-\sum_{i=1}^{n} \Delta_{i} \psi\right) \prod_{i=1}^{n} \frac{\left(\Delta_{i} \psi\right)^{k_{i}}}{k_{i} !} \text { at } \sum_{i=1}^{n} k_{i} \xi_{i}
$$

It follows that

$$
\left\|\gamma_{\pi}\right\|=\exp \left(-\sum_{i=1}^{n} \Delta_{i} \psi\right) \sum_{k_{i} \geq 0} \exp \bar{\omega}\left(\sum_{i=1}^{n} k_{i} \xi_{i}\right) \prod_{i=1}^{n} \frac{\left(\Delta_{i} \psi\right)^{k_{i}}}{k_{i} !} .
$$

Now by assumption (h-ii) there exists an $M>0$ such that $\exp [\bar{\omega}(\xi)] \leq M$ for $0 \leq \xi \leq 2$. Hence, in general, $\exp [\bar{\omega}(\xi)] \leq M^{\xi}$ for all $\xi \geq 1$. Thus we obtain

$$
\log \left\|\gamma_{\pi}\right\| \leq \int_{0}^{1} \exp [\bar{\omega}(\xi)] d \gamma_{\pi}(\xi)+\sum_{i=1}^{n}\left(M^{\xi_{i}}-1\right) \Delta_{i} \psi
$$

Finally, since $\int_{0}^{1} \exp [\bar{\omega}(\xi)] d \gamma_{\pi}(\xi) \leq M$, and since $\int_{0}^{1}\left(M^{\xi}-1\right) d \psi<\infty$, the approximating $\gamma_{\pi}$ 's can be chosen to satisfy the conditions of Lemma 2.2. It follows that $\gamma_{2} \in \subseteq(\bar{\omega})$. Likewise $\int_{1}^{\infty}[\exp (\lambda \xi)-1] d \psi$ can be approximated by sums of the type

$$
\sum_{i=1}^{n}\left[\exp \left(\lambda \xi_{i}\right)-1\right] \Delta_{i} \psi
$$

uniformly in every finite subset of $R(\lambda) \leq 0$. Hence $\gamma_{3}$ can also be approximated by distributions of the type $\gamma_{\pi}$. Now

$$
\bar{\omega}\left(\sum_{i=1}^{n} k_{i} \xi_{i}\right) \leq \sum_{i=1}^{n} k_{i} \bar{\omega}\left(\xi_{i}\right)
$$

so that in this case we have

$$
\log \left\|\gamma_{\pi}\right\| \leq \sum_{i=1}^{n}\left[\exp \bar{\omega}\left(\xi_{i}\right)-1\right] \Delta_{i} \psi
$$

Since $\int_{1}^{\infty}(\exp [\bar{\omega}(\xi)]-1) d \psi<\infty$, it follows as above that $\gamma_{3} \in \mathcal{S}(\bar{\omega})$. 
the conditions (25), and

$$
\lim _{\delta \rightarrow 0+} \sup _{\xi \geq 0}[\bar{\omega}(\xi)-\bar{\omega}(\xi+\delta)]<\infty
$$

then

$$
t^{-1} \log \Phi[\lambda, \gamma(t, \cdot)]=m \lambda+\int_{0}^{\infty}[\exp (\lambda \xi)-1] d \psi,
$$

where $m \geq 0, \psi(\xi)$ is monotone nondecreasing with

$$
\int_{0}^{1} \xi d \psi<\infty, \text { and } \quad \int_{1}^{\infty} \exp [\bar{\omega}(\xi)] d \psi<\infty
$$

It is clear that the continuity conditions (iii) and (iv) of (25) imply (28) by Lemma 2.2. Hence we may avail ourselves of the results of Lemma 3.1. It remains therefore only to verify the statement $\int_{1}^{\infty} \exp [\bar{\omega}(\xi)] d \psi<\infty$. By the assumption (32), there exists a $\Delta>0$ and a $k>0$ such that $\bar{\omega}(\xi+\delta) \geq \bar{\omega}(\xi)-k$ for all $\xi \geq 0$ and all $0<\delta \leq \Delta$. It follows by an induction argument that $\bar{\omega}(\xi+\delta) \geq \omega(\xi)-\left(\delta \Delta^{-1}+1\right) k$ for all $\xi \geq 0$ and all $\delta>0$. Suppose now that $\gamma(\xi)$ is of bounded variation on $[0, \infty)$ and that $\gamma(\xi-m) \in \mathcal{S}(\bar{\omega})$. Then

$$
\int_{0}^{\infty} \exp [\bar{\omega}(\xi)]|d y| \leq \exp \left[\left(m \Delta^{-1}+1\right) k\right] \int_{0}^{\infty} \exp [\bar{\omega}(\xi+m)|d y|
$$

so that $\gamma(\xi) \in \subseteq(\bar{\omega})$. Thus without loss of generality we may assume that $m=0$. For $t$ equal to, say, one, we define $\gamma_{2}$ and $\gamma_{3}$ as before, so that

$$
\log \Phi\left(\lambda, \gamma_{2}\right)=\int_{0}^{1}[\exp (\lambda \xi)-1] d \psi
$$

and

$$
\log \Phi\left(\lambda, \gamma_{3}\right)=\int_{1}^{\infty}[\exp (\lambda \xi)-1] d \psi
$$

Then

$$
\begin{aligned}
\|\gamma\|=\left\|\gamma_{2} * \gamma_{3}\right\|= & \int_{0}^{\infty} \int_{0}^{\infty} \exp \left[\bar{\omega}\left(\xi_{2}+\xi_{3}\right)\right] d \gamma_{2}\left(\xi_{2}\right) d \gamma_{3}\left(\xi_{3}\right) \\
& \geq \int_{0}^{\infty} \int_{0}^{\epsilon} \exp \left[\bar{\omega}\left(\xi_{2}+\xi_{3}\right)\right] d \gamma_{2}\left(\xi_{2}\right) d \gamma_{3}\left(\xi_{3}\right)
\end{aligned}
$$


whence

$$
\|\gamma\| \geq \exp \left[-\left(\epsilon \Delta^{-1}+1\right) k\right]\left\|\gamma_{3}\right\| \gamma_{2}(\epsilon) .
$$

Since $\gamma_{2}$ is a distribution function, we have $\gamma_{2}(\epsilon)>0$ for some $\epsilon>0$. Hence $\gamma_{3} \in S^{2}(\bar{\omega})$. Finally we see that

$$
\Phi\left(\lambda, \gamma_{3}\right)=\exp \left[-\int_{1}^{\infty} d \psi\right] \sum_{n=0}^{\infty} \frac{\left(\Phi\left(\lambda, \gamma_{4}\right)\right)^{n}}{n !},
$$

where $\gamma_{4}(\xi)=0$ for $0 \leq \xi \leq 1$ and $\gamma_{4}(\xi)=\psi(\xi)-\psi(1)$ for $\xi \geq 1$. In other words,

$$
\gamma_{3}=\exp \left[-\int_{1}^{\infty} d \psi\right] \sum_{n=1}^{\infty} \frac{\left(\gamma_{4}^{n *}\right)}{n !}
$$

and in particular $\gamma_{3}(\sigma) \geq \exp \left[-\int_{1}^{\infty} d \psi\right] \gamma_{4}(\sigma)$. Since $\gamma_{3} \in \mathcal{G}(\bar{\omega})$, it follows that $\gamma_{4} \in \Xi(\bar{\omega})$, and hence that

$$
\int_{1}^{\infty} \exp [\bar{\omega}(\xi)] d \psi<\infty
$$

We summarize the results of this section in the following theorem.

THEOREM 3.3. If $\alpha(t, \xi)$ is a semigroup of elements in $\subseteq(\omega)$ satisfying the condition (25), and $\omega(\xi)$ satisfies $\left(h^{*}\right)$, then for $R(\lambda) \leq \omega_{0}$ we have

$$
t^{-1} \log \Phi[\lambda, \alpha(t, \cdot)]=m \lambda+\int_{0}^{\infty}\left(\exp \left[\left(\lambda-\omega_{0}\right) \xi\right]-1\right) d \psi(\xi)+a
$$

where $m \geq 0, a$ is real, and $\psi(\xi)$ is a monotone nondecreasing function such that

$$
\int_{0}^{1} \xi d \psi<\infty \text { and } \int_{1}^{\infty} \exp \left[\omega(\xi)-\omega_{0} \xi\right] d \psi<\infty
$$

Conversely, if $\Phi[\lambda, \alpha(t, \cdot)]$ satisfies (33), then $\alpha(t, \cdot) \in \Xi(\bar{\omega})$ and satisfies (25) for any $\omega(\xi)$ satisfying $(\mathrm{h})$.

Before we conclude this section, a remark is in order about the continuity of the semigroup $\alpha(t, \cdot)$. If we consider $A_{\alpha(t, \cdot)}$ to be a semigroup of operators on $\{(\omega)$, then the strong convergence of these operators to the identity operator 
as $t \longrightarrow 0+$ implies, by Theorem 2.3 , that the continuity hypothesis (25)-(iii) and - (iv) will be satisfied by $\alpha(t, \cdot)$. We shall show in Corollary 4.1 that the converse is likewise true.

4. Semigroups of transformations. In this section, we shall make use of the Banach algebra $S(\omega)$ to develop an operational calculus for the infinitesimal generators of semigroups of transformations on a Banach space to itself. Hille [4, Chap. 15] first introduced such a calculus. The novel feature of the present discussion is that this calculus is used to obtain other semigroups of transformations, and as a consequence to obtain other infinitesimal generators. This method of generating new semigroups has previously been considered in a general way by N. P. Romanoff [10] and in connection with stochastic processes by S. Bochner [2].

Let $\mathfrak{X}$ be a complex Banach space, let $\mathscr{E}(\mathfrak{X})$ be the algebra of linear bounded transformations on $\chi$ to itself, and let $T(t)$ be a semigroup of operators on $[0, \infty)$ to $\mathbb{E}(\mathfrak{X})$ (see [4]) satisfying the following hypothesis:

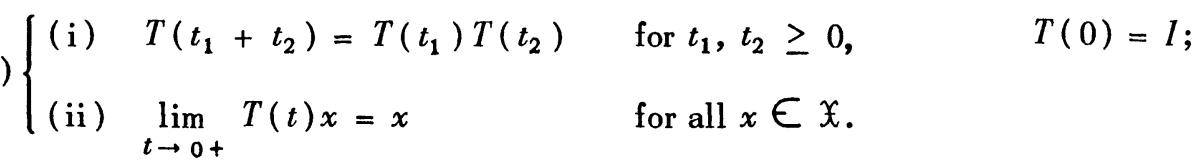

Such a semigroup of transformations will have a closed linear infinitesimal generator $A$ with domain $\mathcal{S}(A)$ dense in $\mathfrak{X}$. It can be shown [4, Theorem 9.4.1] that $T(t)$ is then strongly continuous for $t \geq 0$, and hence that $\|T(t)\|$ is lower semicontinuous. It is clear that the subadditive function

$$
\omega(\xi)=\log \|T(\xi)\|
$$

satisfies the conditions (h) and may be used to define a Banach algebra of the type $\subseteq(\omega)$. For $\alpha(\xi) \in \widetilde{S}(\omega)$, the relation

$$
\Theta(\alpha) x \equiv \int_{0}^{\infty} T(\xi) x d \alpha(\xi)
$$

defines a linear bounded transformation in $\mathscr{E}(X)$. Further, it is easily seen that

$$
\begin{aligned}
& \Theta(e)=I, \\
& \Theta(a \alpha+b \beta)=a \Theta(\alpha)+b \Theta(\beta), \\
& \Theta(\alpha * \beta)=\Theta(\alpha) \Theta(\beta), \\
& \|\Theta(\alpha)\| \leq\|\alpha\| .
\end{aligned}
$$


Hence $\Theta(\alpha)$ is a continuous homeomorphism of $\mathbb{S}(\omega)$ into $\mathscr{E}(X)$ which takes the unit $e$ into the identity $l$. This mapping can be thought of as defining an operational calculus for the infinitesimal generator $A$ of $T(t)$ (see [4] and [9]).

Suppose now that $\alpha(t, \cdot) \in \mathcal{S}(\omega)$ form a semigroup of set functions such that $A_{\alpha(t, \cdot)}$ considered as operators on $\mathcal{l}(\omega)$ satisfy the postulates $(H)$. Then

$$
S(t)=\Theta[\alpha(t, \cdot)]
$$

is clearly a semigroup of operators on $\mathfrak{X}$ to itself by (36). We show next that $S(t)$ converges strongly to $l$ as $t \longrightarrow 0+$. For this purpose we need the following lemma.

Lemma 4.1. Let $A_{\alpha_{n}}$ be a sequence of operators on $\{(\omega)$ which converge strongly to 0 . Suppose $G(\xi) \in\{(\omega) *$ is such that

$$
\lim _{\delta \rightarrow 0+} \sup _{\xi \geq 0}|G(\xi+\delta)-G(\xi)| \exp [-\omega(\xi)]=0 .
$$

Then

$$
\lim _{n \rightarrow \infty} \int_{0}^{\infty} G(\xi) d \alpha_{n}(\xi)=0
$$

By hypothesis,

$$
\lim _{n \rightarrow \infty} \int_{0}^{\infty} G(\xi)\left[\int_{0}^{\xi} f(\xi-u) d \alpha_{n}(u)\right] d \xi=0
$$

for each $f \in \mathcal{L}(\omega)$. By the Fubini theorem,

$$
\int_{0}^{\infty} G(\xi)\left[\int_{0}^{\xi} f(\xi-u) d \alpha(u)\right] d \xi=\int_{0}^{\infty}\left[\int_{u}^{\infty} G(\xi) f(\xi-u) d \xi\right] d \alpha(u)
$$

If we choose $f_{\delta}(\xi)=\delta^{-1}$ for $0 \leq \xi \leq \delta$ and $=0$ elsewhere, then

$$
\int_{0}^{\infty} \delta^{-1}\left[\int_{u}^{u+\delta} G(\xi) d \xi\right] d \alpha_{n}(u) \rightarrow 0
$$

for each $\delta>0$ as $n \longrightarrow \infty$. On the other hand, by (38),

$$
\left|\delta^{-1} \int_{u}^{u+\delta} G(\xi) d \xi-G(u)\right| \leq \exp [\omega(u)] \epsilon(\delta),
$$

where $\epsilon(\delta) \rightarrow 0$ with $\delta$. Hence 


$$
\left|\int_{0}^{\infty} \delta^{-1}\left[\int_{u}^{u+\delta} G(\xi) d \xi\right] d \alpha_{n}(u)-\int_{0}^{\infty} G(u) d \alpha_{n}(u)\right| \leq\left\|\alpha_{n}\right\| \epsilon(\delta),
$$

which converges to zero as $\delta \longrightarrow 0$ uniformly in $n$. It follows that the order of limits may be inverted and hence that (39) is valid.

It is clear that $G(\xi)=x^{*}[T(\xi) x]$ satisfies the condition (38) and belongs to $\left\{(\omega)^{*}\right.$. Further, by hypothesis, the $A_{\alpha(t, \cdot)}$ converge strongly on $\{(\omega)$ to $A_{e}=I$ as $t \longrightarrow 0+$. Hence, by the lemma,

$$
\lim _{t \rightarrow 0+} x^{*}[S(t) x]=\lim _{t \rightarrow 0+} \int_{0}^{\infty} x^{*}[T(\xi) x] d \alpha(t, \xi)=x^{*}(x)
$$

for all $x \in \mathfrak{X}$ and $x^{*} \in \mathfrak{X}^{*}$. The desired strong convergence now follows by a theorem due to Hille [5, p. 93, footnote]. This concludes the proof of the following result.

THEOREM 4.1. If $T(t)$ is a semigroup of operators on $\mathfrak{X}$, and $\alpha(t, \cdot) \in$ $\widetilde{S}(\omega)$ is a semigroup of operators on $\{(\omega)(\omega(\xi)=\log \|T(\xi)\|)$, both satisfying the postulates $(\mathrm{H})$, then $S(t)=\Theta[\alpha(t, \cdot)]$ is again a semigroup of operators on $\mathfrak{X}$ satisfying $(\mathrm{H})$.

If we limit ourselves to semigroups in $\subseteq(\omega)$ of the type studied in $\S 3$, we are then able to obtain a representation for the infinitesimal generator of the semigroup $S(t)=\Theta[\alpha(t, \cdot)]$. To this end, we now prove a generalization of a theorem due to K. Yosida [11, Lemma 2].

THE OREM 4.2. Let $\Re$ be a strongly closed abelian subalgebra of $(\mathfrak{X}(\mathfrak{X})$. For each integer $n$, let $S_{n}(t)$ be a semigroup of transformations in $\Re$ satisfying (H) with infinitesimal generator $B_{n}$. We further assume that $\lim _{n \rightarrow \infty} B_{n}(x)=$ $B^{\prime}(x)$ for a dense subset $\mathscr{S} \subset \cap \supseteqq\left(B_{n}\right)$, and that there exists an $M<\infty$ such that $\left\|S_{n}(t)\right\| \leq M$ for $t \in[0,1]$ and all $n$. Then the $S_{n}(t)$ converge strongly to a semigroup of transformations $S(t)$ in $\Re$ satisfying $(H)$ with infinitesimal generator $B \supset B^{\circ}$.

In the proof of this theorem we make use of a technical device due to Dunford and Segal [3]. For $x \in \mathscr{D} \subset \cap \supseteqq\left(B_{n}\right)$ it is easy to verify that $S_{n}(t-\tau) S_{m}(\tau) x$ is strongly differentiable with respect to $\tau$. Hence

$$
\begin{aligned}
S_{m}(t) x-S_{n}(t) x & =\int_{0}^{t} \frac{d}{d \tau}\left[S_{n}(t-\tau) S_{m}(\tau) x\right] d \tau \\
& =\int_{0}^{t} S_{n}(t-\tau) S_{m}(\tau)\left[B_{m} x-B_{n} x\right] d \tau
\end{aligned}
$$


It follows from our hypothesis that $\left\|S_{n}(t)\right\| \leq M^{1+t}$, and thus that

$$
\left\|S_{m}(t) x-S_{n}(t) x\right\| \leq M^{1+t} \cdot t\left\|B_{n}(x)-B_{m}(x)\right\|
$$

Since the $B_{n}(x)$ form a Cauchy sequence, so do the $S_{n}(t) x$. We define the limit to be $S(t) x$. Now for $x \in \mathcal{D}, S(t) x$ is the uniform limit of continuous functions in every finite interval, and hence is itself continuous. Further, since $\mathscr{P}$ is dense in $\mathfrak{X}$, and because of the uniform boundedness of the $\left\|S_{n}(t)\right\|$ for each $t$, it follows that $S_{n}(t) x \rightarrow S(t) x$ for all $x \in \mathfrak{X}$ and that $\|S(t)\| \leq M^{1+t}$. It is a simple matter to verify that the $S(t)$ form a semigroup of transformations. Also $S(t) x$ is strongly continuous on the dense set $\mathscr{P}$, for $t \geq 0$ and $\|S(t)\| \leq$ $M^{1+t}$ implies that $S(t)$ is strongly continuous on $\mathfrak{X}$ for $t \geq 0$. Thus $S(t)$ satisfies (H). Finally, for $x \in \mathcal{D}$ we have $S_{n}(t) x=x+\int_{0}^{t} S_{n}(\xi) B_{n} x d \xi$ and $S_{n}(\xi) B_{n} x \rightarrow S(\xi) B^{\prime} x$ pointwise and boundedly. Hence

$$
S(t) x=x+\int_{0}^{t} S(\xi) B^{\prime} x d \xi
$$

Thus, for $x \in D$ we have $d(S(t) x) / d t=B^{\prime} x$, and hence $\mathscr{D} \subset \mathscr{D}(B)$ and $B x=B^{\prime} x$ for $x \in$ ?.

We shall hereafter consider only semigroups of functions $\alpha(t, \xi)$ (of bounded variation in every finite interval) whose Laplace transforms $\Phi[\lambda, \alpha(t, \cdot)]$ take the form

$$
\begin{array}{r}
t^{-1} \log \Phi[\lambda, \alpha(t, \cdot)]=m \lambda+\int_{0}^{\infty}\left(\exp \left[\left(\lambda-\omega_{0}\right) \xi\right]-1\right) d \psi(\xi)+a \\
\left(R(\lambda) \leq \omega_{0}\right),
\end{array}
$$

where $m \geq 0, a$ is real, and $\psi(\xi)$ is a monotone nondecreasing function such that

$$
\int_{0}^{1} \xi d \psi<\infty \text { and } \int_{0}^{\infty} \exp \left[\omega(\xi)-\omega_{0} \xi\right] d \psi<\infty
$$

By Theorem 3.3 we see that $\alpha(t, \cdot) \in \mathcal{S}(\omega)$ and satisfies (25). The converse will likewise be valid if $\omega(\xi)$ satisfies (32) in addition to the conditions (h).

THEOREM 4.3. Suppose that $T(\xi)$ is a semigroup of transformations satisfying $(\mathrm{H})$, that $\omega(\xi)=\log \|T(\xi)\|$, and that $\alpha(t, \cdot)$ is a semigroup of elements in $S(\omega)$ having Laplace transforms of the type (40). Then the semigroup of transformations $S(t)=\Theta[\alpha(t, \cdot)]$ satisfies the hypothesis $(\mathrm{H})$, and the 
domain of its infinitesimal generator $B$ contains $\mathscr{I}(A)$. For $x \in \mathscr{}(A)$ we have

$$
B x=m A x+\int_{0}^{\infty}\left[\exp \left(-\omega_{0} \xi\right) T(\xi) x-x\right] d \psi+a x
$$

Let $\alpha_{\epsilon}(t, \cdot)$ be defined as above by means of the same $m$ and $a$ as $\alpha(t, \cdot)$, but with $\psi_{\epsilon}(\xi)=\psi(\epsilon)$ for $\xi \leq \epsilon$ and $\psi_{\epsilon}(\xi)=\psi(\xi)$ for $\xi>\epsilon$. Then, by Theorem 3.3, $\alpha_{\epsilon}(t, \cdot)$ is likewise a semigroup in $\subseteq(\omega)$ satisfying the conditions (25). In the notation of $\S 3$ we have

$$
\left\|\alpha_{\epsilon}(t, \cdot)\right\|_{\omega}=\left.\left\|\beta_{\epsilon}(t, \cdot)\right\|\right|_{\bar{\omega}}=\exp (a t)\left\|\gamma_{\epsilon}(t, \cdot)\right\|_{\bar{\omega}},
$$

where $\bar{\omega}(\xi)=\omega(\xi)-\omega_{0} \xi$, and

$$
t^{-1} \log \Phi\left[\lambda, \gamma_{\epsilon}(t, \cdot)\right]=m \lambda+\int_{\epsilon}^{\infty}(\exp (\lambda \xi)-1) d \psi
$$

Arguing as in Theorem 3.1, we see that

$$
\begin{aligned}
t^{-1} \log \left(\left\|\gamma_{\epsilon}(t, \cdot)\right\| \bar{\omega}\right) \leq \bar{\omega}(m) & +\left(\int_{0}^{1}\left(M^{\xi}-1\right) d \psi+M\right) \\
& +\int_{1}^{\infty}[\exp \bar{\omega}(\xi)-1) d \psi
\end{aligned}
$$

Hence for some $K>0$ we have $\left\|\alpha_{\epsilon}(t, \cdot)\right\|_{\omega} \leq \exp (K t)$ independent of $\epsilon>0$. Now $\psi_{\epsilon} \in S(\bar{\omega})$. Hence

$$
\gamma_{\epsilon}^{\prime}(t, \cdot) \equiv \exp \left(-t \int_{0}^{\infty} d \psi_{\epsilon}\right)\left[\sum_{n=0}^{\infty} \frac{\psi_{\epsilon}^{n^{*}} t^{n}}{n !}\right]
$$

is continuous with respect to $t$ in the $\mathscr{S}(\omega)$ topology, and a fortiori in the strong topology over $\mathfrak{\Omega}(\omega)$. Since the shift semigroup ${ }^{1} e_{m} t$ is likewise strongly continuous, the result holds also for their product $\gamma_{\epsilon}(t, \cdot)=\gamma_{\epsilon}^{\prime}(t, \cdot) * e_{m t}$. We next set

$$
S_{\epsilon}(t) x=\int_{0}^{\infty} T(\xi) x d \alpha_{\epsilon}(t, \xi)=\exp (a t) \int_{0}^{\infty} \exp \left(-\omega_{0} \xi\right) T(\xi) x d \gamma_{\epsilon}(t, \xi)
$$

so that $S_{\epsilon}(t) x$ is given equivalently by

${ }^{1}$ The shift semigroup is defined by $\left(A_{e_{m} t} f\right)(\xi)=f(\xi-m t)$; here $e_{m t}(\xi)=0$ for $\xi \leq m t$ and $=1$ for $\xi>m t$. 


$$
\begin{aligned}
\exp (a t) \int_{0}^{\infty} \exp \left(-\omega_{0} \xi\right) T(\xi) d e_{t m}(\xi) \int_{0}^{\infty} & \exp \left(-\omega_{0} \xi\right) T(\xi) x d \gamma_{\epsilon}^{\prime}(t, \xi) \\
& =\exp \left[\left(a-\omega_{0} m\right) t\right] T(m t) S_{\epsilon}^{\prime}(t) x
\end{aligned}
$$

where

$$
S_{\epsilon}^{\prime}(t) x=\int_{0}^{\infty} \exp \left(-\omega_{0} \xi\right) T(\xi) x d \gamma_{\epsilon}^{\prime}(t, \xi)
$$

Since $\gamma_{\epsilon}^{\prime}(t, \xi)$ is uniformly continuous in $\subseteq(\omega), S_{\epsilon}^{\prime}(t)$ is likewise uniformly continuous, and hence $d S_{\epsilon}^{\prime}(t) / d t$ exists in the uniform topology. Thus

$$
B_{\epsilon}^{\prime} x=\int_{0}^{\infty}\left[\exp \left(-\omega_{0} \xi\right) T(\xi) x-x\right] d \psi_{\epsilon}(\xi)
$$

and $\mathscr{I}\left(B_{\epsilon}^{\prime}\right)=\mathfrak{X}$. Since $S_{\epsilon}(t)$ is the product of two commutative semigroups both strongly convergent to the identity as $t \rightarrow 0$, the same is true of $S_{\epsilon}(t)$; and further, for $x \in \mathscr{S}(A)$,

$$
\begin{aligned}
\left(S_{\epsilon}(\delta) x-x\right) / \delta=S_{\epsilon}^{\prime}(\delta)[T(m \delta) x-x] / \delta+\left(S_{\epsilon}^{\prime}(\delta) x-x\right) / \delta & \\
& \longrightarrow m A x+B_{\epsilon}^{\prime} x \equiv B_{\epsilon} x .
\end{aligned}
$$

Thus $\mathscr{P}\left(B_{\epsilon}\right) \supset \mathscr{P}(A)$, which is dense in $\mathfrak{X}$. Finally, for $x \in \mathscr{S}(A)$,

$$
\exp \left(-\omega_{0} t\right) T(t) x-x=\exp \left(-\omega_{0} t\right) \int_{0}^{t} T(\xi) A x d \xi+\left(\exp \left(-\omega_{0} t\right)-1\right) x
$$

and hence $\left\|\exp \left(-\omega_{0} t\right) T(t) x-x\right\|=O(t)$ as $t \rightarrow 0$. It follows that

$$
\begin{aligned}
B_{\epsilon}^{\prime} x=\int_{\epsilon}^{\infty}\left[\exp \left(-\omega_{0} \xi\right) T(\xi) x-x\right] d \psi & \\
& \longrightarrow \int_{0}^{\infty}\left[\exp \left(-\omega_{0} \xi\right) T(\xi) x-x\right] d \psi \equiv B^{\circ} x,
\end{aligned}
$$

and that

$$
B_{\epsilon} x \rightarrow B x=m A x+\int_{0}^{\infty}\left[\exp \left(-\omega_{0} \xi\right) T(\xi) x-x\right] d \psi
$$


Finally, $\left\|S_{\epsilon}(t)\right\| \leq\left\|\alpha_{\epsilon}(t, \cdot)\right\|_{\omega} \leq \exp (K t)$. It now follows from Theorem 4.2 that there exists a semigroup of transformations $U(t)$ satisfying $(H)$ and with infinitesimal generator $B$ such that $S_{\epsilon}(t) x \rightarrow U(t) x$ for all $x \in X$. It remains to show that $U(t) \equiv S(t)$.

Let us apply the result obtained thus far to $X=\{(\omega)$ and $(T(t) f)(u)=$ $f(u-t)$. The semigroup $T(t)$ satisfies (H). Now we know that the integral

$$
\int_{0}^{\infty} T(\xi) f d \alpha_{\epsilon}(t, \xi)=\int_{0}^{u} f(u-\xi) d \alpha_{\epsilon}(t, \xi)
$$

converges in $\mathfrak{X}$ to $U(t) f$. On the other hand, it follows from Lemma 2.2 that $\lim _{\epsilon \rightarrow 0} \alpha_{\epsilon}(t, \xi)=\alpha(t, \xi)$ for each point of continuity of $\alpha(t, \xi)$. Thus if $f(u)$ is continuous and differs from zero only on a finite interval then

$$
\int_{0}^{u} f(u-\xi) d \alpha_{\epsilon}(t, \xi) \rightarrow \int_{0}^{u} f(u-\xi) d \alpha(t, \xi)
$$

pointwise and hence in norm. Since this is true on a dense subset in $\Omega(\omega)$, and since $\left\|\alpha_{\epsilon}(t, \cdot)\right\| \leq \exp (K t)$, it follows that $A_{\alpha_{\epsilon}}(t, \cdot)$ converges strongly to $A_{\alpha}(t, \cdot)$. Finally, if we apply Lemma 4.1 once again with

$$
G(\xi)=x^{*}[T(\xi) x] \in\{(\omega) *,
$$

we obtain

$$
x^{*}\left[S_{\epsilon}(t) x\right] \longrightarrow \int_{0}^{\infty} x^{*}[T(\xi) x] d \alpha(t, \xi)=x^{*}[S(t) x]
$$

It follows that $S(t) \equiv U(t)$. This concludes the proof of Theorem 4.3.

As a corollary we obtain a partial converse to Theorem 2.3.

COROLLARY 4.1. If $\alpha(t, \cdot)$ is a semigroup of elements in $\mathbb{S}(\omega)$ satisfying

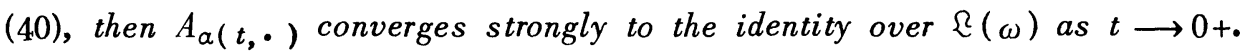

In terms of the previous theorem, we set $\mathfrak{X}=\mathfrak{\Omega}(\omega)$ and $(T(t) f)(u)=$ $f(u-t)$, a right translation in $\mathfrak{L}(\omega)$. Then

$$
S(t) f=\int_{0}^{\infty} T(\xi) f d \alpha(t, \xi)=\int_{0}^{u} f(u-\xi) d \alpha(t, \xi)=A_{\alpha(t, \cdot)} f .
$$

Hence by Theorem 4.3, $A_{a(t, \cdot)}$ converges strongly to the identity as $t \longrightarrow 0+$.

We conclude this section with a discussion of the spectrum of the infinitesimal generator $B$ of the semigroup $S(t)$. We shall need the following lemma. 
Lemma 4.2. Let $\alpha(t, \cdot)$ be a semigroup in $\subseteq(\omega)$ such that the operators $A_{\alpha(t, \cdot)}$ on $\left\{(\omega)\right.$ satisfy the hypothesis $(\mathrm{H})$ and let $\omega^{\prime}(t)=\log \|\alpha(t, \cdot)\|$. Suppose further that $G(\xi) \in\left\{(\omega)^{*}\right.$ satisfies the condition (38). Then for $g(t) \in\left\{\left(\omega^{\prime}\right)\right.$ we have

(41) $\int_{0}^{\infty} g(t)\left[\int_{0}^{\infty} G(\xi) d_{\xi} \alpha(t, \xi)\right] d t=\int_{0}^{\infty} G(\xi) d_{\xi}\left(\int_{0}^{\infty} g(t) \alpha(t, \xi) d t\right)$, where

$$
\int_{0}^{\infty} g(t) \alpha(t, \xi) d t \equiv \int_{0}^{\infty} g(t) A_{\alpha}(t, \cdot) d t
$$

converges in the strong topology over $\{(\omega)$.

For $f \in\{(\omega), \alpha(t, \cdot) * f$ is continuous for $t \geq 0$ in the $\{(\omega)$ topology. Hence the integral of $g(t)$ times a bounded linear functional on $\alpha(t, \cdot) * f$ is equal to the functional on $\left(\int_{0}^{\infty} g(t) \alpha(t, \cdot) d t\right) * f$; that is,

$$
\begin{aligned}
\int_{0}^{\infty} G(\xi)\left[\int_{0}^{\infty} f(\xi-u) d u\left(\int_{0}^{\infty} g(t) \alpha(t, u) d t\right)\right] d \xi \\
=\int_{0}^{\infty} g(t)\left[\int_{0}^{\infty} G(\xi)\left(\int_{0}^{\infty} f(\xi-u) d_{u} \alpha(t, u)\right) d \xi\right] d t
\end{aligned}
$$

Fubini's theorem permits the interchange of the $u$ and $\xi$ integrations, so that

$$
\begin{aligned}
\int_{0}^{\infty}\left[\int_{0}^{\infty} G(\xi) f(\xi-u) d \xi\right] d_{u}\left(\int_{0}^{\infty} g(t) \alpha(t, u) d t\right) \\
\quad=\int_{0}^{\infty} g(t)\left[\int_{0}^{\infty}\left(\int_{0}^{\infty} G(\xi) f(\xi-u) d \xi\right) d_{u} \alpha(t, u)\right] d t
\end{aligned}
$$

Again we set $f_{\delta}(\xi)=\delta^{-1}$ for $0 \leq \xi \leq \delta$ and $=0$ elsewhere. Then, as in Lemma 4.1,

$$
\left|G(\xi) f_{\delta}(\xi-u) d \xi-G(u)\right| \leq \exp [\omega(u)] \epsilon(\delta),
$$

where $\epsilon(\delta) \rightarrow 0$ with $\delta$. Hence, taking the limit in (42) as $\delta \rightarrow 0$, we obtain (41).

THЕОВЕМ 4.4. Given the semigroup of operators $T(t)$ satisfying $(\mathrm{H})$ with infinitesimal generator $A$, let $\Sigma(A)$ be the spectrum of $A$ and let $\Sigma(B)$ be the spectrum of the infinitesimal generator $B$ of the semigroup $S(t)=\Theta[\alpha(t, \cdot)]$. If the Laplace transform of $\alpha(t, \cdot)$ satisfies $(40)$, then 


$$
\Sigma(B) \supset \Phi_{1}[\Sigma(A)]
$$

where

$$
\Phi_{1}(\lambda)=m \lambda+\int_{0}^{\infty}\left(\exp \left[\left(\lambda-\omega_{0}\right) \xi\right]-1\right) d \psi(\xi)+a
$$

In the proof of this theorem we make use of material developed in an earlier paper [9]. It was there shown that if $\lambda_{0} \in \Sigma(A)$ and $\alpha \in \Subset(\omega)$, then

$$
\int_{0}^{\infty} \exp \left(\lambda_{0} \xi\right) d \alpha \in \Sigma[\Theta(\alpha)]
$$

Now let $R(B ; \lambda)$ be the resolvent of $B$, and set $\omega^{\prime}(t)=\log \|\alpha(t, \cdot)\|$. Then for $R(\lambda)>\omega_{0}^{\prime}=\inf \omega^{\prime}(t) / t$, we have [4, Theorem 11.6.1]

$$
R(B, \lambda)=\int_{0}^{\infty} \exp (-\lambda t) S(t) d t
$$

Hence for $x \in \mathfrak{X}$ and $x^{*} \in \mathfrak{X}^{*}$ we obtain

$$
x^{*}[R(B ; \lambda) x]=\int_{0}^{\infty} \exp (-\lambda t)\left[\int_{0}^{\infty} x^{*}[T(\xi) x] d_{\xi} \alpha(t, \xi)\right] d t
$$

We now apply Lemma 4.2 with

$$
G(\xi)=x^{*}[T(\xi) x] \in\left\{(\omega)^{*} \text { and } g(t)=\exp (-\lambda t) \in\left\{\left(\omega^{\prime}\right)\right.\right.
$$

The right side of the above equation can then be written as

$$
\int_{0}^{\infty} x *[T(\xi) x] d \xi \quad\left[\int_{0}^{\infty} \exp (-\lambda t) \alpha(t, \cdot) d t\right]
$$

where

$$
\int_{0}^{\infty} \exp (-\lambda t) \alpha(t, \cdot) d t \equiv \int_{0}^{\infty} \exp (-\lambda t) A_{\alpha}(t, \cdot) d t
$$

converges in the strong operator topology in $\subseteq(\omega)$ over $\{(\omega)$. Since this holds for all $x \in \mathfrak{X}$ and $x^{*} \in \mathfrak{X}^{*}$, we have

$$
R(B ; \lambda)=\int_{0}^{\infty} T(\xi) d_{\xi}\left[\int_{0}^{\infty} \exp (-\lambda t) \alpha(t, \cdot) d t\right]
$$

It follows, for $\lambda_{0} \in \Sigma(A)$ and $R(\lambda)>\omega_{0}^{\prime}$, that 


$$
\int_{0}^{\infty} \exp \left(\lambda_{0} \xi\right) d_{\xi}\left[\int_{0}^{\infty} \exp (-\lambda t) \alpha(t, \cdot) d t\right] \in \Sigma[R(B ; \lambda)]
$$

Now if $\mu=\left\{(\omega)^{*}\right.$, it follows from general integration theory that

$$
\mu\left\{\left[\int_{0}^{\infty} \exp (-\lambda t) \alpha(t, \cdot) d t\right]^{*} f\right\}=\int_{0}^{\infty} \exp (-\lambda t) \mu\left[\alpha(t, \cdot)^{*} f\right] d t
$$

for all $f \in \Omega(\omega)$. In particular, let $\mu(f)=\int_{0}^{\infty} \exp \left(\lambda_{0} \xi\right) f(\xi) d \xi$, where $R\left(\lambda_{0}\right) \leq \omega_{0}$; then $\mu\left(\alpha^{*} f\right)=\Phi(\lambda, \alpha) \mu(f)$. Hence,

$$
\begin{aligned}
{\left[\int_{0}^{\infty} \exp \left(\lambda_{0} \xi\right) d \xi\left(\int_{0}^{\infty} \exp (-\lambda t) \alpha(t, \cdot) d t\right)\right] \mu(f) } \\
=\int_{0}^{\infty} \exp (-\lambda t) \Phi\left[\lambda_{0}, \alpha(t, \cdot)\right] \mu(f) d t .
\end{aligned}
$$

For some $f \in \Omega(\omega)$ we have $\mu(f) \neq 0$, so that

$$
\begin{aligned}
\int_{0}^{\infty} \exp \left(\lambda_{0} \xi\right) d \xi & \left(\int_{0}^{\infty} \exp (-\lambda t) \alpha(t, \cdot) d t\right) \\
& =\int_{0}^{\infty} \exp (-\lambda t) \Phi\left(\lambda_{0}, \alpha(t, \cdot)\right) d t=\left[\lambda-\Phi_{1}\left(\lambda_{0}\right)\right]^{-1}
\end{aligned}
$$

We conclude that

$$
\left[\lambda-\Phi_{1}\left(\lambda_{0}\right)\right]^{-1} \in \Sigma[R(B, \lambda)]
$$

It now follows from Theorem 3.1 of [9] that $\Phi_{1}\left(\lambda_{0}\right) \in \Sigma(B)$.

\section{REFERENCES}

1. S. Banach, Théorie des opérations linéaires, Warsaw, 1932.

2. S. Bochner, Diffusion equation and stochastic processes, Proc. Nat. Acad. Sci. U.S.A. 35 (1949), $368-370$.

3. N. Dunford and I. E. Segal, Semi-groups of operators and the Weierstrass theorem, Bull. Amer. Math. Soc. 52 (1946), 911 - 914.

4. Einar Hille, Functional analysis and semi-groups, Amer. Math. Soc. Colloquium Publ. 31, New York, 1948.

5. Soc. 56 (1950), $89-114$.

6. A. Khintchine, Deduction nouvelle d'une formule de M. Paul Lévy, Bull. Univ. d'état à Moscou. Série Int., Sect. A. 1(1937), 1-5. 
7. A. Kolmogoroff, Sulla forma generale di un processo stocastico omogeneo, Atti. Accad. Naz. Lincei, series 6, 15 (1932), 805-808, 866-869.

8. Paul Lévy, Théorie de l'addition des variables aléatoires, Monographies des probabilitiés, fasc. I, Paris, 1937.

9. R. S. Phillips, Spectral theory for semi-groups of linear transformations, Trans. Amer. Math. Soc. 71 (1951), 393-415.

10. N. P. Romanoff, On one parameter groups of linear transformations I, Ann. of Math., Series 2, 48 (1947), 216-233.

11. Kôsaku Yosida, An operator-theoretical treatment of temporally homogeneous Markoff processes, J. Math. Soc. Japan, 1 (1949), 244- 253.

The University of Southern California

Los Angeles, California 



\title{
PACIFIC JOURNAL OF MATHEMATICS
}

\section{EDITORS}

\section{R. M. RobINSON \\ University of California \\ Berkeley 4, California}

\author{
*R. P. Dilwor TH \\ California Institute of Technology \\ Pasadena 4, California
}

\author{
E. F. BECKENBACH, Managing Editor \\ University of California \\ Los Angeles 24, California
}

${ }^{*}$ During the absence of Herbert Busemann in 1952.

\section{ASSOCIATE EDITORS}

$\begin{array}{llll}\text { R. P. DILWORTH } & \text { P. R. HALMOS } & \text { B } \emptyset \text { RGE JESSEN } & \text { J. J. STOKER } \\ \text { HERBERT FEDERER } & \text { HEINZ HOPF } & \text { PAUL LÉVY } & \text { E. G. STRAUS } \\ \text { MARSHALL HALL } & \text { R. D. JAMES } & \text { GEORGE PÓLYA } & \text { KÖSAKU YOSIDA }\end{array}$

\section{SPONSORS}

UNIVERSITY OF BRITISH COLUMBIA

CALIFORNIA INSTITUTE OF TECHNOLOGY

U NIVERSITY OF CALIFORNIA, BERKELEY

UNIVERSITY OF CALIFORNIA, DAVIS

UNIVERSITY OF CALIFORNIA, LOS ANGELES

UNIVERSITY OF CALIFORNIA, SANTA BARBARA

OREGON STATE COLLEGE

UNIVERSITY OF OREGON

\author{
UNIVERSITY OF SOUTHERN CALIFORNIA \\ STANFORD UNIVERSITY \\ WASHINGTON STATE COLLEGE \\ UNIVERSITY OF WASHINGTON \\ AMERICAN MATHEMATICAL SOCIETY \\ NATIONAL BUREAU OF STANDARDS, \\ INSTITUTE FOR NUMERICAL ANALYSIS
}

Mathematical papers intended for publication in the Pacific Journal of Miathematics should be typewritten (double spaced), and the author should keep a complete copy. Manuscripts may be sent to any of the editors. All other communications to the editors should be addressed to the managing editor, E. F. Beckenbach, at the address given above.

Authors are entitled to receive 100 free reprints of their published papers and may obtain additional copies at cost.

The Pacific Journal of Mathematics is published quarterly, in March, June, September, and December, by the University of California, Berkeley 4, California. The price per volume (4 numbers) is $\$ 8.00$; single issues, $\$ 2.50$. Special price to individual faculty members of supporting institutions and to individual members of the American Mathematical Society: $\$ 4.00$ per volume; single issues, $\$ 1.25$.

Subscriptions, orders for back numbers, and changes of address should be sent to the publishers, University of California Press, Berkeley 4, California.

Printed at Ann Arbor, Michigan. Entered as second class matter at the Post Office, Berkeley, California.

\section{UNIVERSITY OF CALIFORNIA PRESS • BERKELEY AND LOS ANGELES}




\section{Pacific Journal of Mathematics}

\section{Vol. 2, No. $3 \quad$ March, 1952}

Lars V. Ahlfors, Remarks on the Neumann-Poincaré integral equation .... 271

Leonard P. Burton, Oscillation theorems for the solutions of linear, nonhomogeneous, second-order differential systems ............ 281

Paul Civin, Multiplicative closure and the Walsh functions . . . . . . . . . . . 291

James Michael Gardner Fell and Alfred Tarski, On algebras whose factor algebras are Boolean .................................. 297

Paul Joseph Kelly and Lowell J. Paige, Symmetric perpendicularity in Hilbert geometries .................................. 319

G. Kurepa, On a characteristic property of finite sets .............. 323

Joseph Lehner, A diophantine property of the Fuchsian groups ......... 327

Donald Alan Norton, Groups of orthogonal row-latin squares ........... 335

R. S. Phillips, On the generation of semigroups of linear operators ....... 343

G. Piranian, Uniformly accessible Jordan curves through large sets of relative harmonic measure zero ........................ 371

C. T. Rajagopal, Note on some Tauberian theorems of $O . S z \tilde{A} ; s z \ldots \ldots \ldots 377$

Halsey Lawrence Royden, Jr., A modification of the Neumann-Poincaré method for multiply connected regions .................... 385

George H. Seifert, A third order irregular boundary value problem and the associated series ...................................... 395

Herbert E. Vaughan, Well-ordered subsets and maximal members of ordered sets....

Hans F. Weinberger, An optimum problem in the Weinstein method for eigenvalues.

Shigeki Yano, Note on Fourier analysis. XXXI. Cesàro summability of Fourier series. 